\title{
Simulation analysis of intelligent scheduling model of large mechanical work task
}

\author{
Rong Xin-yan, Li Zeng-xin, Zhang Li-yan \\ (Qinhuangdao Institute of Technology; Hebei Qinhuangdao 066100 China)
}

Keywords: large mechanical work task; optimal standard; scheduling conflict; intelligent scheduling; local particle optimization theory

\begin{abstract}
As the complexity of the task of large mechanical work continues to increase, during the assembly scheduling process between each sub task, direct optimal standard of local scheduling have conflicts, contradiction of different sub task direct scheduling process is becoming more and more prominent. When the traditional intelligent scheduling method faces complicated mechanical work, is easy to be affected by scheduling contradiction between many local jobs, which makes the scheduling method fall in convergence, it is difficult to form an optimal scheduling scheme to meet the demand of all scheduling. This paper presents intelligent scheduling method for tasks of large mechanical work based on adding particle planning. The local particle optimization theory is introduced in the process of scheduling, the sub scheduling process is regarded as set to obtain the best solution for the order in the collection. Assigning intelligent scheduling process for tasks of large mechanical work reasonably, the most reasonable scheduling method is selected to realize optimal planning of task scheduling. The experimental results show that, algorithm used for assembly sequence planning in complex processes, can effectively avoid the conflict between the scheduling processes, and improve the efficiency of assembly execution.
\end{abstract}

\section{Introduction}

The sub tasks scheduling in large task [1-2], is the core problem being researched in the manufacturing process, and widely concerned by many scholars [3]. At this stage, the large mechanical work task scheduling methods mainly include the method based on ant colony algorithm, repeated game algorithm and time domain scheduling model [4]. Among them, the large mechanical work task scheduling method based on time domain scheduling model is a sub task scheduling method used in manufacturing of high frequency [6-9]. The above method is used for assembly scheduling, assuming mechanical task complexity is relatively high, some the conventional sequencing process is very difficult to form the optimal scheduling ratio, will cause being lack of scheduling mechanism of the optimal solution when many tasks are implementing simultaneously, thus affecting the efficiency of assembly, unable to obtain the ideal complex process assembly scheduling results [10].

Aiming at the limitation of the traditional algorithm, a large mechanical work task intelligent scheduling method based on particle planning algorithm is proposed. During the complex process of the assembly, according to the related theory of particle planning algorithm to schedule assembly task of complex working procedures, so as to select the optimal way of assembly sequence planning. Experimental simulation is processed for assembly problems in the complex working procedures, the experimental simulation results show that assembly scheduling in the complex process with this algorithm can optimize the assembly index, make machinery and task scheduling are more reasonable, and effectively improve the efficiency of assembly.

\section{Intelligent scheduling principle of large mechanical working tasks}

The relationship between each sub task in large mechanical work task is more complex, and needs reasonable scheduling process. The principle is as follows: $K$ sub tasks of strong independence are sorted reasonably, so that scheduling efficiency of each sub task reaches the maximum value. The data set constituted of scheduling tasks can be described by the following 
formula:

$$
\left\{\begin{array}{l}
K=\left\{K_{1}, K_{2}, \cdots, K_{p}\right\} \\
Q=\left\{Q_{1}, Q_{2}, \cdots, Q_{n}\right\}
\end{array}\right.
$$

In the formula, $U_{j}$ is the $j$-th sub task, $Q_{k}$ is the state parameters in $k$-th large mechanical work. The execution time expectation matrix of sub task $K_{j}$ of large mechanical work in the process of scheduling can be described with $F_{U D, j k}$, the matrix is as follows:

$$
F_{U D, p \times n}=\left|\begin{array}{llc}
F_{U D, 11} & \cdots & F_{U D, 1 n} \\
\vdots & \vdots & \vdots \\
F_{U D, p 1} & \cdots & F_{U D, p n}
\end{array}\right|
$$

According to the matrix, the earliest finish time of $K_{j}$ is $F_{U D, j k}$. If the matrix is transformed, the following results can be obtained:

$$
F_{D U, p \times n}=\left|\begin{array}{ccc}
F_{D U, 11} & \cdots & F_{D U, 1 n} \\
\vdots & \vdots & \vdots \\
F_{D U, p 1} & \cdots & F_{D U, p n}
\end{array}\right|
$$

In the formula, $F_{D U, j k}$ is the time required for execution of all mechanical tasks. The discriminant formula of whether the $K_{j}$ state is $Q_{k}$ is as follow:

$$
y_{j k}=\left\{\begin{array}{l}
1, \text { The status of assembly task } K_{j} \text { is } Q_{k} \\
0, \quad \text { otherwise }
\end{array}\right.
$$

The constraint conditions of large mechanical work task scheduling can be described with the following formula:

$$
\left\{\begin{array}{l}
\sum_{j=1}^{p} y_{j k}=1, j \in\{1,2, \cdots, p\} \\
\sum_{k=1}^{n} y_{j k}=1, k \in\{1,2, \cdots, n\}
\end{array}\right.
$$

\section{The method of assembly sequence planning based on particle planning algorithm}

\section{Principle of particle scheduling algorithm}

In the particle scheduling algorithm, each subject has the initial spatial position parameters and velocity, the fitness function corresponding to the particle is used to calculate the performance. The optimal solution for large mechanical working task scheduling problem is corresponding to and the target in the search space, each particle have to obtain the search object according to the initial space position parameters and the running speed, finally acquire the optimal solution of the problem.

In the particle scheduling algorithm, the optimal solution can be calculated according to the target function of particle. Assuming that the target function value is small, the particles have higher adaptability. The particle's serial number is ${ }^{j}$, then the initial spatial position of the particle can be calculated with the following formula:

$$
Y_{j}(u)=\left(y_{j 1}(u), y_{j 2}(u), \cdots, y_{j E}(u)\right)
$$

In the process of iterative processing, optimal solution needs to be tracked with particle, so as to obtain updated speed and updated spatial position parameters of the particles, the two update formula are as follows:

$$
\begin{aligned}
& W_{j l}(u+1)=x W_{j l}(u)+d_{1} s_{1}\left(Q_{j l}(u)-Y_{j l}(u)\right) \\
& Y_{j l}(u+1)=Y_{j u}(u)+W_{j l}(u+1)
\end{aligned}
$$

In the formula, $x$ is the weight coefficient of the particle, $d_{1}$ and $d_{2}$ are acceleration in the running process of particle, in general, $d_{1}=d_{2}=1, s_{1}$ and $s_{2}$ are integers less than $1, l=1,2, \cdots, e$.

\section{The sub task optimal particle scheduling method}

The number of sub tasks in mechanical task is $K$, the numbers of tasks included in each sub task is $Q$, the amount of mechanical tasks is $N$, particle is consisted of two $K \times Q$ dimensional vector $Y_{Q}$ and $Y_{N}$. 
According to the objective function, the fitness function corresponding to all particles can be calculated. Solving the objective function, can acquire the start time and end time of scheduling sequence in complex mechanical work task. The objective functions are as follows:

$$
\min \left(U\left(K_{n}\right)=\min \left\{\max \left[U_{1}, U_{2}, \cdots, U_{n}\right\}\right.\right.
$$

The following formula is utilized to describe the adaptive function of particles:

$$
g_{j}=\frac{100 \times o p t}{U_{j}\left(K_{n}\right)}
$$

In the formula, $U_{j}\left(K_{n}\right)$ is the time needed after executing ${ }^{j}$-th mechanical task, opt is optimal solution searched in specified space.

Assuming the scheduling sequence in large mechanical work task has reached the maximum number of iterative processing or the optimal solution repeatedly reaches the preset value, then stop the operation of iterative processing, to obtain the optimal solution. Through the method stated above, according to the related theory of particle scheduling algorithm, to have the optimal planning of scheduling sequence in large mechanical work tasks, so as to obtain ideal planning results.

\section{Experimental simulation}

In order to evaluate the effectiveness of the proposed particle scheduling algorithm in the intelligent scheduling of large mechanical work tasks, there is the need for an experiment. In the experimental process, sub scheduling experiment simulation is done with MATLAB. The experimental objects can be described by the following figure:

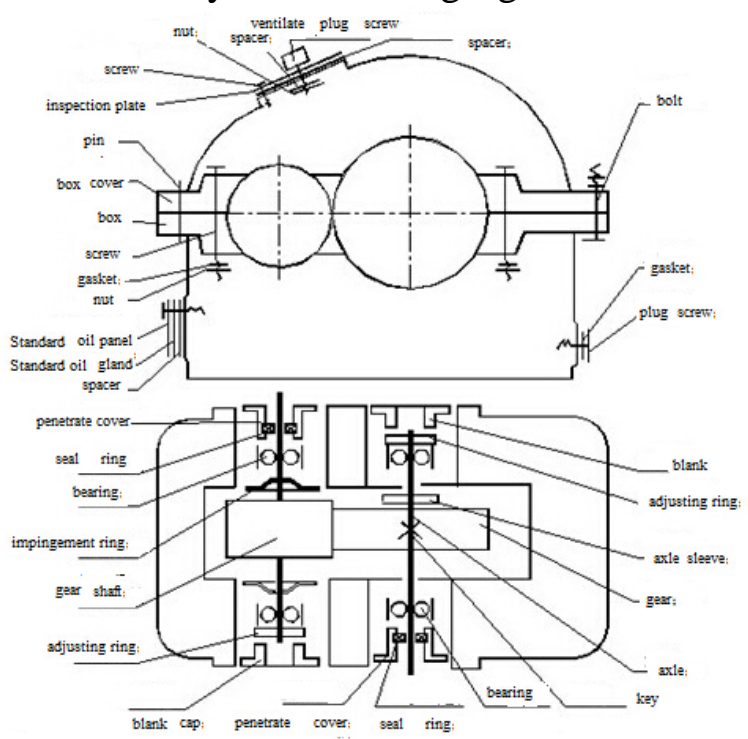

Figure 1 the object of the simulation experiment

With the traditional algorithm and the proposed algorithm for large mechanical work task scheduling simulation experiment, under the same experimental conditions, the scheduling results obtained with two different algorithms are compared. The number of experiments for each experiment is 200 times, the average value of 200 experiments is calculated as experimental results.

When the proposed algorithm is utilized for large mechanical work task intelligent scheduling optimization processing, the initial parameters need to be set. The specific data can be described by the following table: 
Table 1 The main parameters of the proposed algorithm

\begin{tabular}{ll}
\hline Name & Value \\
\hline The number of particles & 500 \\
The number of machines & 100 \\
The number of tasks & 300 \\
The penalty factor & 0.84 \\
The training factor & 3 \\
The maximum of iterative processing & 200 \\
times & \\
\hline
\end{tabular}

The complex mechanical sub tasks are divided, the value range of acquired sub task is $[10,300]$, the value range of time required for completing each sub assembly task is ${ }^{[1,30]}$. Using the above algorithm for mechanical work task scheduling, the condition for iteration stop is when the iterative processing times reached the maximum value or completion time of consecutive 40 sub task are basically same.

The most important factor influencing mechanical work task complexity is the number of sub tasks. When the sub task number is 100 and 200, the experimental simulation results can be described by figure 2 and figure 3 :

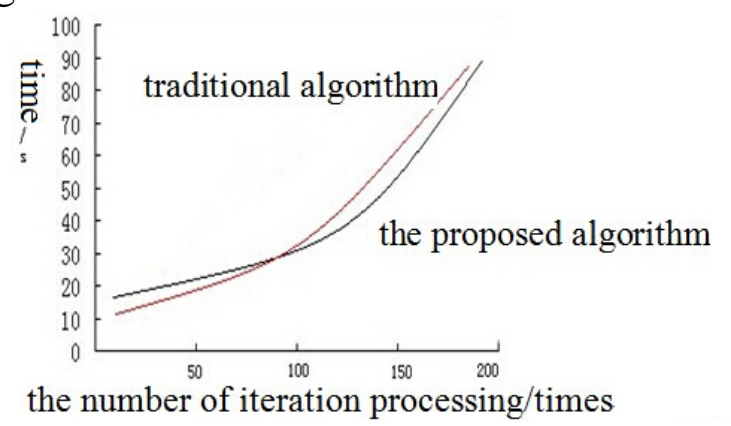

Figure 2 the experimental simulation results when sub task is 100

According to the above figure, when the sub task is 100, both the traditional algorithm and the proposed algorithm are utilized for large mechanical work task intelligent scheduling, required sub task execution time are basically same. With the increase of the number of iterative processing, required time for intelligent scheduling with the proposed algorithm is slightly shorter than the traditional algorithm.

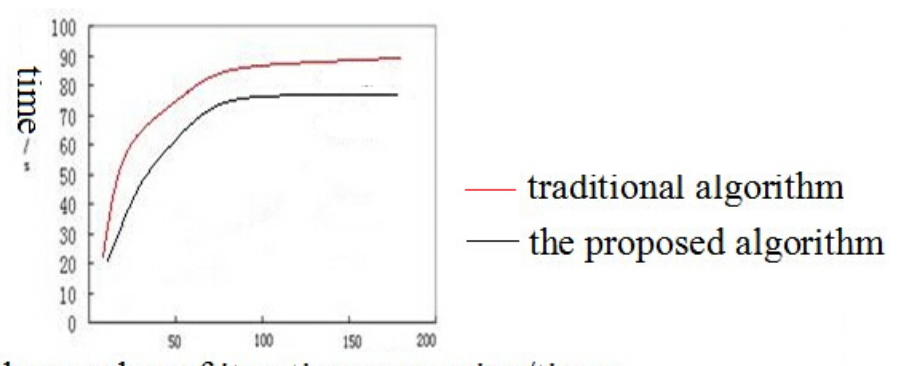

the number of iteration processing/times

Figure 3 the experimental simulation results when sub task is 200

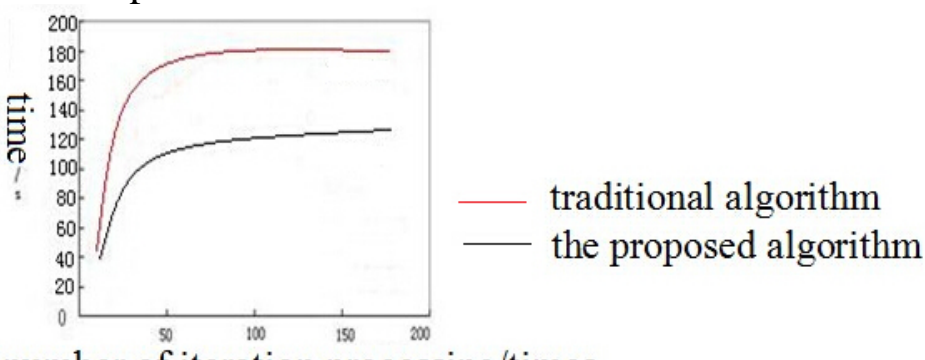

the number of iteration processing/times

Figure 4 the experimental simulation results when sub task is 300 
When the number of tasks reached 200, large mechanical work task intelligent scheduling with the traditional algorithm, takes 20s longer than the proposed algorithm. When the number of tasks reached 300, with the traditional algorithm takes 70 s longer than the proposed algorithm. Based on the above experimental contents, it can be learned that, in the situation of high complex relationship between sub tasks, with this algorithm for large mechanical work task intelligent scheduling, the time took by the scheduling process is much shorter than the traditional algorithm, fully demonstrate the advantages of the proposed algorithm.

\section{Conclusions}

Aiming at the defects at efficiency of large mechanical work task intelligent scheduling of traditional approach, a mechanical task scheduling method based on particle algorithm is provided. The related theory of particle scheduling algorithm is elaborated in detail, provide a theoretical basis for sequence scheduling of sub tasks based on particle scheduling algorithm. The particle scheduling method adopted for scheduling among each sub task in large mechanical work tasks, the most reasonable dispatching mode is selected, so as to achieve the optimal scheduling for working sequence of sub tasks. The experimental results show that, for the task of large mechanical work of intelligent scheduling by using this algorithm, can effectively improve the execution efficiency of intelligent scheduling, so as to improve the production efficiency.

\section{References}

[1] Zheng Genrang. Traffic congestion scheduling scheme based on hybrid artificial fish swarm algorithm [J]. Computer simulation, 2012.6:328-331.

[2] CHIEN A, CALDER B, ELBERT S, et al. Entropia: Architecture and Performance of an Enterprise Desktop Grid System [J]. Journal of Parallel and Distributed Computing, 2003, 63(5):597-610.

[3] ROCHWERGER B, BREITGAND D, LEVY E, et al. The Reservoir Model and Architec -ture for Open Federated Cloud Computing [J]. IBM Journal of Research and Development, 2009, 53(4):1-17.

[4] ALI S, SIEGEL H J, MAHESWARAN M, et al. Representing Task and Machine Heterogen eities for Heterogeneous Computing systems [J]. Journal of Science and Engineering, 2000, 3(3): 195-207.

[5] Li N, SUN DB, ZOU T. An Analysis for a Particle's Trajectory of PSO based on Difference Equation [J]. Chinese Journal of Computers, 2006, 29(11):2052- 2061.

[6] RATNAWEERA A, HALGAMUGE SK, Watson HC. Self-Organizing Hierarchical Particle Swarm Optimizer with Time-varying Acceleration Coefficients [J]. IEEE Trans. on Evolutionary Computation, 2004, 8(3):240- 255.

[7] Liu Lidong, Cai Huai. Hybrid ant colony algorithm fused into genetic algorithm [J]. Computer engineering and design, 2008, 29(5): 1248-1249.

[8] Zhong Qiuxi, Chen Huowang. The design of genetic operators in task matching and scheduling [J]. Journal of National University of Defense Technology, 2000, 22(3):34-38.

[9] FOSTER I, KESSELMAN C, TUECKE S.The Anatomy of the Grid: Enabling Scalable Virtual Organization [J]. High-Performance Computing Applications, 2001, 15(3): 200-222.

[10] Yuan Lulai, Zeng Guosun, Jiang Lili, et al. Dynamic level scheduling based on trust model in Grid Environment [J]. Chinese Journal of Computers, 2006, 29(7):1217-1224. 